\title{
DISPROOF OF THE CONJECTURED SUBEXPONENTIALITY OF CERTAIN FUNCTIONS IN PERCOLATION THEORY
}

\author{
J. VAN DEN BERG,* Delft University of Technology
}

\begin{abstract}
Consider bond-percolation on a graph $G$ with sites $S(G)$. We disprove the conjecture of Hammersley (1957) that the function $n \rightarrow \sup _{s \in S(G)} E$ [the number of sites $s^{\prime}$ at distance $n$ from $s$ which can be reached from $s$ by an open path which, except for $s^{\prime}$, only passes through sites at distance smaller than $n$ from $s$ ] is always subexponential.
\end{abstract}

\section{Introduction}

Percolation theory has been introduced by Broadbent and Hammersley (1957). For a recent introduction to the subject see Kesten (1982), Chapter 1.

Let $G$ be a locally finite graph (i.e. the number of bonds incident to any site is finite) and denote the set of sites of $G$ by $S(G)$. Let the bonds of $G$, independent of each other, be open with probability $p$ and closed with probability $1-p$. The length of a path is the number of bonds it contains. The distance between two sites is the length of the shortest path which connects them. Define, for $s \in S(G)$ :

$N^{n}(s)$ is the set of sites at distance $\leqq n$ from $s$.

$B^{n}(s)$ is the set of sites at distance $n$ from $s$.

$E_{n}(s)$ is the expected number of sites $s^{\prime} \in B^{n}(s)$ for which there exists an open path from $s$ to $s^{\prime}$ which, except for $s^{\prime}$, only passes through sites in $N^{n-1}(s)$.

Finally, define $F_{n}=\sup _{s \in S(G)} E_{n}(s)$.

Though $E_{n}$ and $F_{n}$ also depend on $p$, we omit this parameter.

Hammersley (1957) conjectured that $F_{n+m} \leqq F_{n} F_{m}$ always. In the next section we show that there exists a case for which $F_{2}>F_{1}^{2}$ so that the conjecture is false.

\section{The counterexample}

Consider, for a positive integer $r$, the graph with $1+r+r^{2}$ sites denoted by $c$; $s_{i}$, $1 \leqq i \leqq r$, and $s_{i, j}, 1 \leqq i, j \leqq r$; and with bonds $\left(c, s_{i}\right), 1 \leqq i \leqq r ;\left(s_{i}, s_{j}\right), 1 \leqq i, j \leqq r, i \neq j$; and $\left(s_{i}, s_{i, j}\right), 1 \leqq i, j \leqq r$. This graph can be imagined as a central site $c$, surrounded by and

Received 18 April 1984.

* Postal address: Department of Mathematics, Delft University of Technology, Julianalaan 132, 2628 BL Delft, The Netherlands.

Research supported by the Netherlands Foundation for Mathematics SMC with financial aid from the Netherlands Organization for the Advancement of Pure Research (ZWO). 
connected with a complete graph on $r$ sites, each of which having a bond to $r$ other sites which have no further connections.

Now consider bond-percolation on this graph with $p$ the probability of a bond to be open. It is clear that, for each site $s, E_{1}(s)$ equals $p$ times the number of bonds incident to $s$ and this is maximal if $s$ is one of the $s_{i}$ 's, in which case it equals $2 r p$. So

$$
F_{1}=2 r p \text {. }
$$

Further, $F_{2}$ is at least $E_{2}(c)$ which, by symmetry, equals the number of sites at distance 2 from $c$ multiplied by the probability of the event that at least one of them, say $s_{11}$, can be reached from $c$ by an open path. (By the structure of the graph the condition of containing no sites, except $s_{11}$, outside $N^{1}(c)$ is automatically fulfilled.) Note that this event occurs if and only if the bond $\left(s_{1}, s_{11}\right)$ is open (which happens with probability $p$ ) and there exists, inside the complete graph on the set $\left\{c, s_{1}, s_{2}, \cdots, s_{r}\right\}$ an open path from $c$ to $s_{1}$. Denote the probability of the latter event by $P(p, r)$. Using independence we get

$$
F_{2} \geqq r^{2} p P(p, r) .
$$

Hence, by" (2.1) and (2.2)

$$
\frac{F_{2}}{F_{1}^{2}} \geqq \frac{P(p, r)}{4 p}
$$

It is easily seen that for fixed $p$

$$
\lim _{r \rightarrow \infty} P(p, r)=1, \quad 0<p \leqq 1 .
$$

Now fix $p$ between 0 and $\frac{1}{4}$. Then, for $r$ sufficiently large, the right-hand side of (2.3) is larger than 1 , in contradiction to the conjecture.

\section{Remarks.}

(i) With the help of the finite graphs above it is easy to obtain a counterexample concerning an infinite connected graph. For example, connecting the site $c$ with an infinite chain does not increase the value $F_{1}$.

(ii) One might think that the conjecture is true if, in the definition of $E^{n}(s)$, all open paths of which all sites are in $N^{n}(s)$ are allowed. However, consider the tree consisting of a site $c$ which is connected with six sites $s_{1}, s_{2}, \cdots, s_{6}$, each $s_{i}$ in its turn being connected with six sites $s_{i, 1}, s_{i, 2}, \cdots, s_{i, 6}$. Add to this tree, for each $j \leqq 6$, the bonds $\left(s_{1, j}, s_{2, j}\right),\left(s_{3, j}, s_{4, j}\right)$ and $\left(s_{5, j}, s_{6, j}\right)$. For the graph thus obtained it is easily verified that $F_{1}=7 p$ and according to the new definition of $E_{n}, F_{2} \geqq E_{2}(c)=36 P$ [there exists an open path from $c$ to $\left.s_{1,1}\right] \geqq 36\left(p^{2}+p^{3}-p^{5}\right)$ which, if $p=\frac{1}{2}$, appears to be larger than $49 p^{2}$.

\section{References}

Broadbent, S. R. and Hammersley, J. M. (1957) Percolation processes. Proc. Camb. Phil. Soc. 53, 629-641; 642-645.

HAMmersley, J. M. (1957) Percolation processes: lower bounds for the critical probability. Ann. Math. Statist. 28, 790-795.

Kesten, H. (1982) Percolation Theory for Mathematicians. Birkhäuser, Boston. 